\title{
Gestação ectópica gemelar com embriões vivos: um relato de caso
}

\author{
Gemelar ectopic pregnancy with live embroys: a case report \\ Embarazo ectópico gemelar com embriones vivos: reporte de un caso
}

Lázaro Luiz de Paula Neto, André Luiz Meles Ferreira1, João Prette Netto², Fernanda Pedezzi Biagi2*.

\section{RESUMO}

Objetivo: Discutir a abordagem adotada pela equipe médica em um caso de gravidez gemelar ectópica com embriões vivos, sendo este um relato raro, com pouca literatura publicada, além de discorrer sobre as características de gestações ectópicas e gemelares, e quais as condutas disponíveis para o seguinte caso. Detalhamento do caso: Relata o caso de uma tercigesta, sem fatores de risco, com gestação ectópica tubária gemelar a esquerda com presença de atividade cardíaca detectável em ambos os embriões. O diagnóstico foi concluído por meio de ultrassonografia transvaginal e realizado laparotomia com salpingectomia como medida terapêutica. Considerações finais: A gravidez ectópica (GE) é uma intercorrência obstétrica cada vez mais frequente, em virtude da maior prevalência de seus fatores de risco, tornando-se, assim, um problema de saúde pública. A GE gemelar unilateral com embriões vivos, é um evento raro, com uma incidência de 1:250.000. A publicação de novos casos possibilita a evolução no entendimento da patologia, sua investigação e terapêuticas.

Palavras-chave: Gravidez ectópica, Gravidez tubária, Gravidez gemelar, Laparotomia.

\begin{abstract}
Objective: Discuss the approach taken by the medical team in a case of ectopic twin pregnancies with live embryos, which is a rare report, with little published literature, in addition to discussing the characteristics of ectopic and twin pregnancies. Case details: Reports the case of a third child, without risk factors, with ectopic twin-tube ectopic pregnancy with the presence of fetal cardiac activity detectable in both embryos. The diagnosis was concluded by means of transvaginal ultrasound where laparotomy with salpingectomy was performed as a therapeutic measure. Final considerations: Ectopic pregnancy (EG) is an increasingly frequent obstetric complication, due to the higher prevalence of its risk factors, thus becoming a public health problem. Unilateral twin GE with live embryos is a rare event, with an incidence of 1:250,000. The publication of new cases allows the evolution in the understanding of the pathology, its diagnostic and therapeutic investigation.
\end{abstract}

Keywords: Ectopic pregnancy, Tubal pregnancy, twin pregnancy, laparotomy.

\section{RESUMEN}

Objetivo: Discutir el abordaje adoptado por el equipo médico en un caso de embarazos gemelares ectópicos con embriones vivos, que es un informe poco común, con poca literatura publicada, además de discutir las características de los embarazos ectópicos y gemelares. Detalle del caso: Relata el caso de un tercero, sin factores de riesgo, con embarazo ectópico ectópico de trompas gemelas ectópico con presencia de actividad cardíaca fetal detectable en ambos embriones. El diagnóstico se concluyó mediante ecografía transvaginal donde se realizó laparotomía con salpingectomía como medida terapéutica. Consideraciones finales: El embarazo ectópico ( $E G$ ) es una complicación obstétrica cada vez más frecuente, debido a la mayor prevalencia de sus factores de riesgo, convirtiéndose así en un problema de salud pública. La GE gemelar unilateral con embriones vivos es un evento raro, con una incidencia de 1:250.000. La publicación de nuevos casos permite la evolución en la comprensión de la patología, su investigación diagnóstica y terapéutica.

Palabras clave: Embarazo ectópico, Embarazo tubárico, Embarazo gemelar, Laparotomía.

${ }^{1}$ Fundação Santa Casa de Misericórdia de Franca, Franca - SP.

2 Universidade de Franca, Franca - SP. *E-mail: ferpbiagi@hotmail.com

SUBMETIDO EM: 3/2021

ACEITO EM: 3/2021

PUBLICADO EM: 5/2021 


\section{INTRODUÇÃO}

A gravidez ectópica (GE) é definida como uma gestação na qual a implantação e o desenvolvimento do concepto ocorrem fora da cavidade uterina, sendo a tuba uterina o local com maior frequência de implantação, cerca de $95 \%$ dos casos (ZUGAIB M, 2019). É a principal causa de mortalidade materna no primeiro semestre de gestação, sendo responsável por 6 a 13\% dos óbitos (FEBRASGO, 2018).

A frequência de casos de GE tem aumentado na atualidade, tanto pela prevalência dos seus fatores de risco, como pela melhora no seu diagnóstico rápido e precoce, sendo responsável por 1 a $2 \%$ das gestações e considerada um problema de saúde pública em alguns países (CRIOLLO RA, et al., 2016; MARTINS EF, et al., 2017).

A ocorrência da GE está associada a fatores relacionados à lesão da tuba uterina ou dificuldade no transporte do concepto até a cavidade. Dentre os fatores de risco desta comorbidade, os mais relevantes são: uso de dispositivo intrauterino (DIU); história prévia ou atual de doença inflamatória pélvica (DIP); cirurgia tubária ou uterina prévia; história anterior de gravidez ectópica; emprego de técnicas de reprodução assistida, em especial a fertilização in vitro; uso de anticoncepção de emergência (pílula do dia seguinte); anormalidade anatômica das trompas; massas extrínsecas que causam compressão tubária; tabagismo; início precoce da atividade sexual; idade materna avançada; múltiplos parceiros sexuais; endocrinopatias; endometriose e raça branca (ZUGAIB M, 2019; FREITAS F, et al., 2011; REZENDE J e MONTENEGRO CAB, 2014; HERRERA GM, et al., 2017; BERNARDES LS, et al., 2018; BEREK JS, 2013).

O quadro clínico clássico ocorre em apenas 50 a $60 \%$ dos casos, sendo caracterizado pela presença de dor abdominal em baixo ventre tipo cólica, de forte intensidade, podendo ser de início súbito ou progressivo, seguida de sangramento vaginal de variável coloração e intensidade, além de atraso menstrual (FREITAS F, et al., 2011; ZUGAIB M, 2019).

Em situações nas quais ocorre rotura da tuba uterina, o quadro torna-se mais grave, com a possível presença de dor abdominal difusa devido ao hemoperitôneo, vômitos, palidez, e outros sinais característicos de choque hemorrágico e instabilidade hemodinâmica (FEBRASGO, 2018). Sendo assim, o diagnóstico precoce é de suma importância para evitar a ocorrência de complicações maternas e o agravo do quadro, diminuindo a incidência de morbidade e mortalidade materna por GE (BEREK JS, 2013; JUNIOR EJ, et al., 2008).

No diagnóstico da gravidez ectópica, o quadro clínico isolado não confirma tal intercorrência obstétrica, sendo necessário realizar exames complementares para confirmação diagnóstica e seguimento. $O$ exame laboratorial mais utilizado é a dosagem da gonadotrofina coriônica humana ( $\beta$-hCG) (FEBRASGO, 2018; BERNARDES LS, et al., 2018).

A ultrassonografia é o exame de imagem utilizado para o diagnóstico, de preferência sendo realizado via transvaginal (USTV), através da qual é possível observar a presença do saco gestacional fora da cavidade uterina e se há presença de líquido na cavidade abdominal (REZENDE J e MONTENEGRO CAB, 2014; FREITAS F, et al., 2011; HOFFMAN BL e SCHORGE JO, 2014).

A conduta na grande maioria dos casos de gravidez ectópica, é a abordagem cirúrgica, sendo a salpingectomia total ou parcial do local de implantação do embrião a cirurgia de escolha. $O$ tratamento conservador com Metotrexato (MTX) ainda é pouco utilizado e deverá obedecer a critérios rígidos para sua aplicação (ZUGAIB M, 2019; FEBRASGO, 2018; CRIOLLO RA, et al., 2016; REZENDE J e MONTENEGRO CAB, 2014; HOFFMAN BL e SCHORGE JO, 2014).

Situações nas quais ocorre a presença simultânea de dois ou mais conceptos no útero, ou fora dele, constituem a chamada gravidez múltipla e representam aproximadamente 1 a $3 \%$ de todas as gestações. (BRASIL, 2012). Esse tipo de gravidez, por sua vez, pode ser classificado de acordo com a quantidade de conceptos (dupla ou gemelar, tripla, etc) ou de ovos que foram fecundados (gestação monozigótica ou dizigótica) (ZUGAIB M, 2019; REZENDE J e MONTENEGRO CAB, 2014; ESCOLA UFRJ, 2015; FEBRASGO, 2014; DUTTA D, 2015). 
Assim como nos casos de GE, houve um aumento significativo do número de gestações múltiplas, em virtude do uso cada vez mais frequente de medicações indutoras da ovulação e do emprego de diferentes técnicas de reprodução assistida, além de outros fatores como: história familiar de gemelaridade, idade materna avançada (maior que 35 anos), raça negra e multiparidade (ZUGAIB M, 2019; ESCOLA UFRJ, 2015; FEBRASGO, 2014; POSSER AO, et al., 1998; BEEN R, et al., 2016; DEDE M, et al., 2008; DUTTA D, 2015).

A gravidez ectópica tubária gemelar (GETG) é uma forma rara de gestação ectópica que possui uma incidência em torno de 1:200. Casos de GETG nas quais é possível observar a presença de embriões vivos são ainda menos frequentes, com taxas de 1:125.000 gestações espontâneas, sendo uma das formas mais raras de gestação gemelar. O seguinte relato descreve o caso de uma GE tubária gemelar unilateral íntegra, com embriões vivos na ultrassonografia, diagnosticada na sexta semana de gravidez, ocorrido no Hospital de Referência de uma cidade no interior do Estado de São Paulo, no ano de 2020 (BERNARDES LS, et al., 2018; POSSER AO, et al., 1998).

O seguinte trabalho tem por objetivo abordar a conduta adotada por profissionais médicos frente a um caso de gravidez gemelar ectópica com embriões vivos, assim como discutir as características de gestações ectópicas e gemelares. Pretende-se ainda elaborar uma análise comparativa da abordagem realizada frente a esse caso, usando como base as principais literaturas e relatos existentes a respeito deste assunto.

\section{DETALHAMENTO DO CASO}

O relato do caso foi autorizado pela paciente em questão, na qual assinou o termo de livre consentimento esclarecido (TCLE) para a divulgação dos seus dados pessoais e médicos, tendo como objetivo, enriquecimento da literatura e exploração de diferentes condutas diante do caso exposto. $O$ trabalho foi submetido ao Conselho de Ética e Pesquisa (CEP) da Santa Casa de Franca-SP, no qual foi aprovado, identificando-se pelo parecer 4.529.737.

Paciente de 36 anos, tercigesta com 2 partos normais anteriores, sem história de aborto ou gestação ectópica prévia, com idade gestacional de seis semanas e quatro dias de acordo com a data da última menstruação (DUM), compareceu ao serviço de Pronto Atendimento de Ginecologia e Obstetrícia com queixa de dor em baixo ventre, negando sangramento ou outras queixas, e, com história negativa de coito nas últimas 24 horas. A gestante negava uso de DIU, infecções sexualmente transmissíveis, doença inflamatória pélvica (DIP), uso de anticoncepção de emergência, cirurgia ginecológica prévia, história familiar de GE ou hábito tabagista.

Ao exame físico apresentava-se em bom estado geral, anictérica, acianótica e afebril, corada e hidratada, com abdome plano, ruídos hidroaéreos presentes e normoativos, ausência de massas ou visceromegalias, sinais de descompressão brusca e Giordano negativos, porém com dor abdominal difusa associada a contratura de defesa voluntária à palpação.

O exame ginecológico especular demonstrou um colo uterino fechado, sem presença de sangramento coletado em fundo de saco ou ativo à manobra de valsava. Durante exame de toque vaginal bimanual, paciente apresentou dor à palpação de fundo de saco posterior.

$\mathrm{O}$ caso foi conduzido inicialmente como uma gestação inicial incipiente e dor abdominal à esclarecer, sendo realizada analgesia e solicitado exames laboratoriais: hemograma, $\beta$-hCG quantitativo, Proteína $C$ Reativa (PCR) e urina tipo 1. O exame de $\beta$-hCG apresentou-se com valor de 9.799 , enquanto os demais exames não demonstraram alterações.

De acordo com a sintomatologia e o resultado dos exames, realizou-se uma ultrassonografia transvaginal (USTV) onde visualizou-se um útero anterovertido com miométrio homogêneo, cavidade endometrial virtual, ovários habituais e fundo de saco com grande quantidade de líquido livre, além da presença de saco gestacional à esquerda em topografia anexial, contendo duas vesículas vitelínicas, com dois embriões vivos em seu interior, com batimentos cardíacos fetais (BCF) presentes. O embrião 1 apresentava um comprimento cabeça-nádega (CCN) de 0,6 cm e BCF de 131 bpm, e o embrião 2 um CCN de 0,7 cm e um BCF de 121 bpm (Figura 1 e 2). Com o resultado da USTV foi possível fechar o diagnóstico de gestação gemelar ectópica rota à esquerda com embriões vivos (Figura 3). 
Figura 1 - Demonstração da atividade cardíaca no embrião 1.

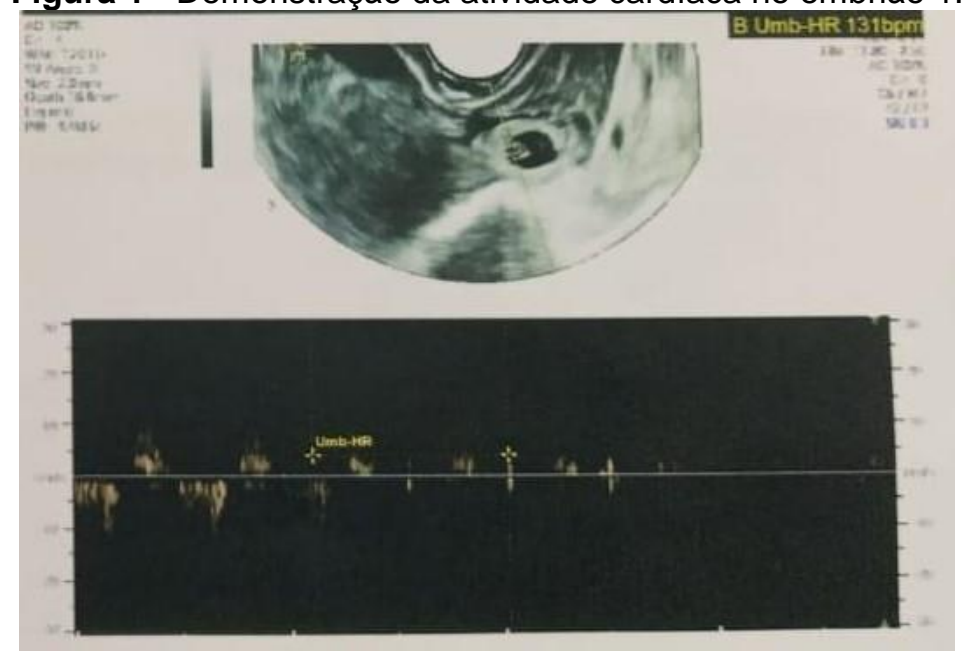

Fonte: Neto LLP, et al, 2021.

Figura 2 - Demonstrção da atividade cardíaca no embrião 2.

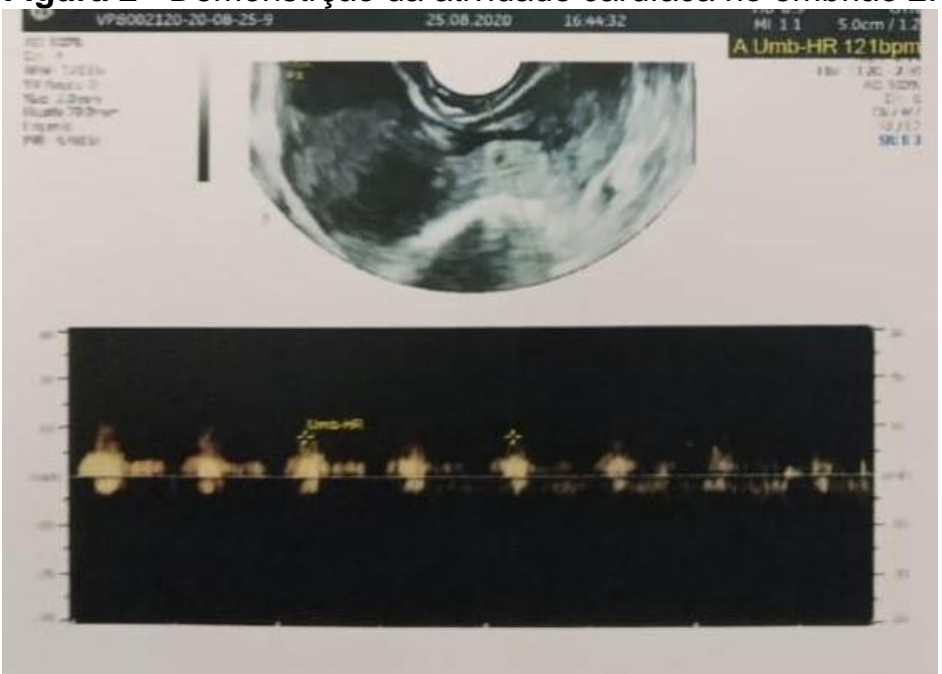

Fonte: Neto LLP, et al, 2021.

Figura 3 - USTV evidenciando a presença de uma gravidez ectópica tubária gemelar unilateral à esquerda.

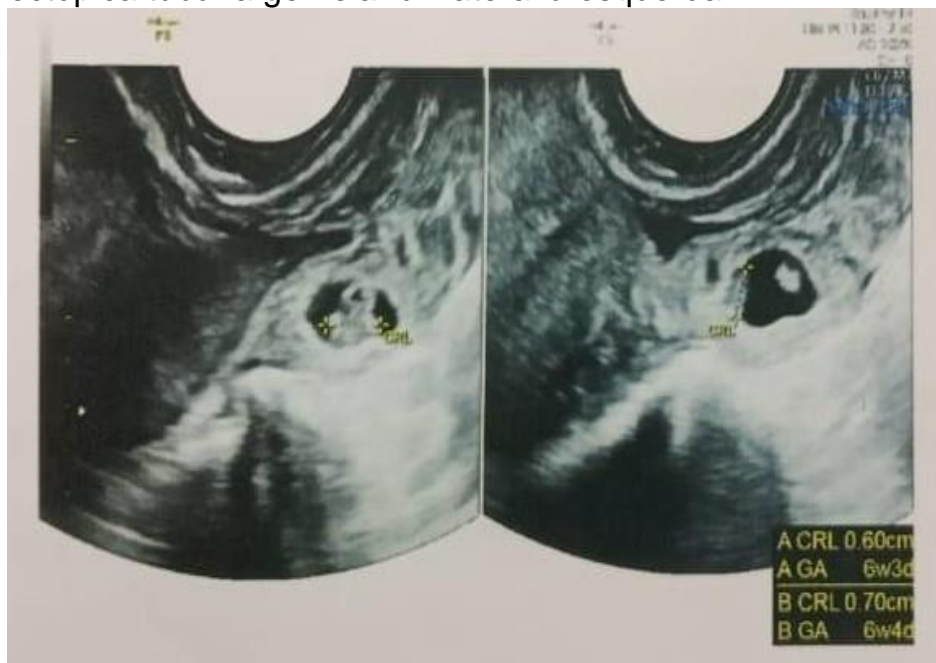

Fonte: Neto LLP, et al, 2021. 
Em seguida, a paciente foi encaminhada para abordagem cirúrgica, sendo realizado laparotomia com salpingectomia à esquerda, sem intercorrências. Durante o procedimento foi encontrado grande quantidade de sangue livre em cavidade abdominal e visualizou-se a GE à esquerda sendo retirada e o material enviado para análise anatomopatológica no qual indicou uma tuba uterina cilíndrica medindo $6,5 \mathrm{~cm}$ de comprimento, com diâmetros variando entre 0,8 a 1,9 cm (Figura 4), com camada serosa pardacenta, congesta e rota com coágulos associados, sendo confirmado o diagnóstico histopatológico de gestação ectópica tubárea. A paciente recebeu alta hospitalar no segundo dia pós-operatório em boas condições clínicas, em uso de sintomáticos e sulfato ferroso, com ferida operatória limpa e sem sinais flogísticos, com abdome flácido, ruídos hidroaéreos presentes com eliminação de flatos e pouco doloroso a palpação profunda em região de hipogástrio.

Figura 4 - À esquerda, laparotomia evidenciando a presença de gravidez ectópica tubária esquerda; à direita, tuba uterina contendo o saco gestacional e embriões retirado e enviado para análise anatomopatológica.

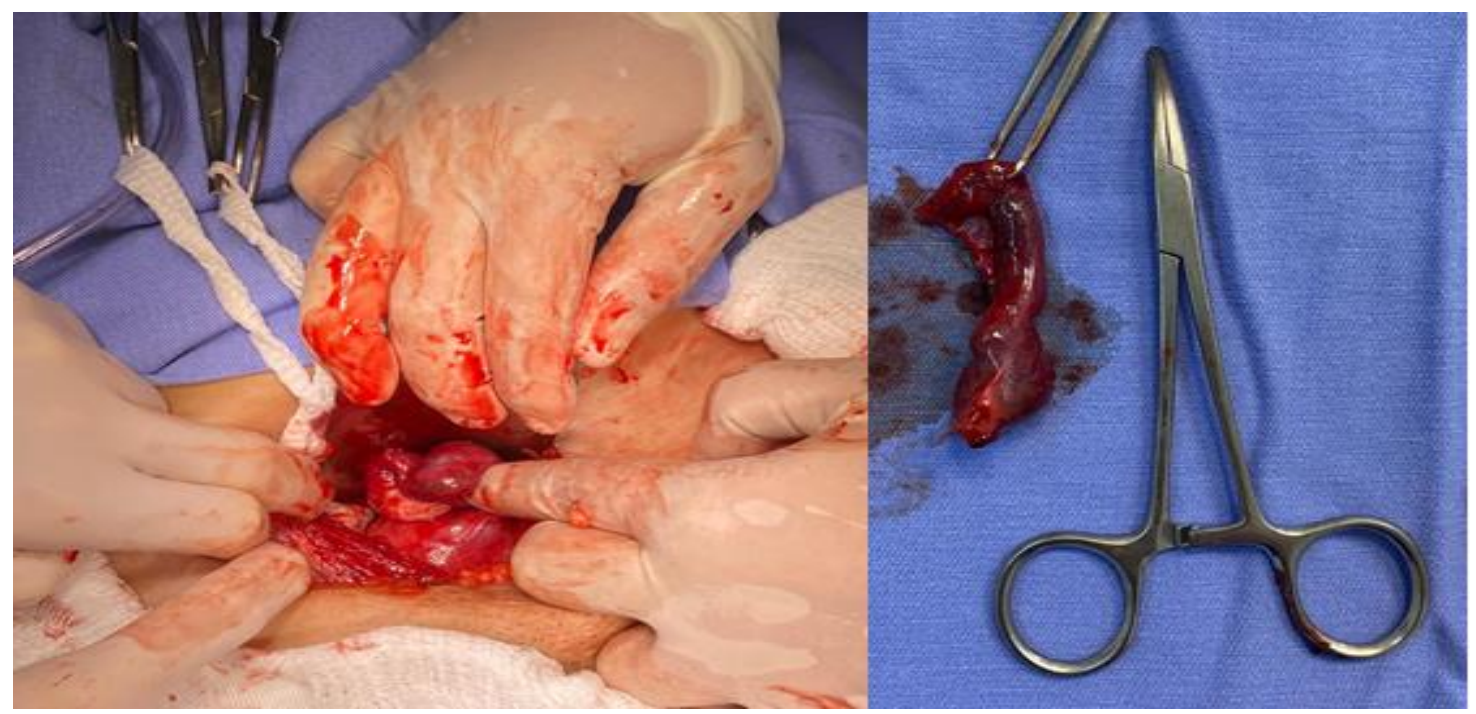

Fonte: Neto LLP, et al, 2021.

\section{DISCUSSÃO}

O caso relatado acima, faz parte dos poucos encontrados na literatura sobre gravidez ectópica tubária gemelar (GETG), uma vez que ambas as situações possuem baixa incidência separadamente, portanto, a ocorrência de ambas na mesma gestação é considerada um evento atípico e raro. O primeiro caso de GETG relatado foi escrito por De Ott D (1891) e posteriormente por Guelandi M, et al. (1994) e os embriões também apresentavam batimentos cardíacos fetais. Desde então, poucos casos são relatados, sendo encontrado também pequenas variações, como gestação ectópica bilateral e casos de gestação uterina e ectópica simultâneas. Dentre essas outras variações relatadas, a GE gemelar unilateral, mesmo rara, acaba sendo a mais prevalente, ocorrendo na proporção de 1: 200 casos de gestação ectópica (ZUGAIB M, 2019; BRASIL, 2012; ESCOLA UFRJ, 2015; VOHRA S, et al., 2014).

Além da raridade da gestação ectópica gemelar, a presença de batimentos cardíacos fetais (BCF) em ambos os embriões merecem considerável atenção, já que na maioria dos casos encontrados na literatura, poucos embriões possuem atividade cardíaca quando a intercorrência obstétrica em questão é diagnosticada. Segundo artigo estrangeiro publicado no Journal Clinical Case Reports no setor de Ginecologia e Obstetrícia em 2014, a ocorrência de BCF em ambos embriões nos casos de GE gemelar possuí prevalência de 13 casos para cada 100 gestações desse tipo (BEEN R, et al., 2016; BAI A, et al., 2014).

Atualmente a gestação ectópica (GE) apresenta crescente incidência em mulheres com ou sem os fatores de risco conhecidos para tal intercorrência obstétrica, tornando-se então um problema de saúde pública, principalmente em países em desenvolvimento, por apresentar grande morbidade e mortalidade, quando o 
diagnóstico não é realizado precocemente (ZUGAIB M, 2019; FEBRASGO, 2018). Conforme o caso narrado e assim como em um relato publicado na Revista de Medicina de Minas Gerais, no qual foi descrito um caso de gravidez ectópica tubária gemelar à esquerda, a paciente deste relato em questão também não possuía nenhum fator de risco descrito na literatura que poderia ser responsável por tal ocorrência clínica (BERNARDES LS, et al., 2018).

Em contrapartida, dois relatos descreveram casos de GE gemelar nos quais ambas pacientes apresentaram fatores de risco para tal condição. No primeiro, descrito por Betti M, et al. (2018), a paciente em questão possuia história de endometriose, bem como de uma gestação ectópica à direita, tratada com laparotomia e salpingectomia, sete meses antes de ser diagnosticada com novo caso de GE gemelar; no caso seguinte, de Pascual MA, et al. (2001) a paciente de 39 anos apresentava antecedentes de dois abortos prévios, tendo realizado terapia de fertilizaçao in vitro antes de engravidar.

O quadro clínico relatado, condiz com os sintomas de GE, porém, sem perdas vaginais (ZUGAIB M, 2019; FEBRASGO, 2018; REZENDE J e MONTENEGRO CAB, 2014). Diante dos sinais e sintomas elaborou-se hipóteses diagnósticas como gestação inicial incipiente, ameaça de aborto e gestação ectópica, sendo solicitado exames complementares para conclusão diagnóstica e abreviar a decisão terapêutica. Ao realizar a USTV constatou-se a presença da GE gemelar à esquerda e líquido em fundo de saco. Diante de tais achados se fez o diagnóstico de GE com rotura tubária contraindicando o tratamento conservador e sendo conduta imediata a resolução da gestação por via cirúrgica. Com laparotomia e salpingectomia, assim como apontado na literatura e nos casos utilizados como base para este trabalho (CRIOLLO RA, et al., 2016; REZENDE J e MONTENEGRO CAB, 2014; HERRERA, GM, et al., 2017; BERNARDES LS, et al., 2018; BRASIL, 2012; POSSER AO, et al., 1998; BAI A, et al., 2014).

Indo de encontro à conduta realizada no relato descrito, as principais literaturas e casos utilizados como referências para este trabalho descrevem relatos nos quais o tratamento cirúrgico foi priorizado, por via laparoscópica com salpingectomia associada. É possível observar que a terapêutica conservadora acaba por ser preterida na maioria dos serviços, e mesmo quando utilizada, se faz necessária posterior abordagem cirúrgica (BEEN R, et al., 2016; POSSER AO, et al., 1998; BETTI M, et al., 2018; PASCUAL MA, et al., 2001).

Em razão da raridade do caso e dos poucos relatos na literatura sobre gestação ectópica gemelar com BCF presente em ambos os embriões, eventos que se somados torna o caso atípico e incomum, optou-se por relatá-lo pela escassez de fontes sobre tratamentos e condutas que poderiam ser tomadas para melhorar a terapêutica de tal intercorrência do primeiro trimestre da gestação. Evidenciou-se com os casos encontrados na literatura e o apresentado que a ocorrência da implantação tubária é a mais comum, e ocorrência de gemelaridade monozigótica nesses casos tem maior predominância. Ambos os relatos citados anteriormente também possuem similaridade na implantação à esquerda, ausência de fatores de risco e na conduta adotada (BERNARDES LS, et al., 2018; BEEN R, et al., 2016; BAI A, et al., 2014).

O aumento no emprego de DIUs e de técnicas de reprodução assistida, bem como o início precoce da atividade sexual, múltiplos parceiros e gravidez em idade avançada trouxeram consigo um aumento da incidência dos casos de gestação ectópica, todavia, é possível observar que tal diagnóstico também deve ser considerado em mulheres sem tais fatores de risco. Além disso, fica evidente que a evolução dos exames complementares disponíveis tornou seu diagnóstico cada vez mais precoce, promovendo a diminuição de complicações e a redução da morbimortalidade materna. $O$ relato apresentado traz consigo a raridade de uma gestação tubária gemelar unilateral com fetos vivos, uma vez que a maioria dos embriões ectópicos não apresentam BCFs quando se faz o diagnóstico de tal intercorrência obstétrica.

\section{REFERÊNCIAS}

1. BAI A, et al. Unilateral Live Tubal Twin Ectopic Pregnancy. J. Clinical Cases Report, 2014; 1(1).

2. BEEN R, et al. Spontaneous Live Twin Ectopic Pregnancy: a Case Report. WINJ, 2016; 3(1).

3. BEREK JS. Gynecology. 15th ed. Philadelphia: Berek D.L, 2013; 1965p. 9

4. BERNARDES LS, et al. Gravidez ectópica tubária gemelar unilateral: relato de caso. Revista de Medicina de Minas Gerais, 2018; 28(5).

5. BETTI M, et al. Unilateral twin tubal pregnancy: a case report and review of the literature. Acta Biomed, 2018; 89(3). 
6. BRASIL. Manual Técnico do Ministério da Saúde para Gestação de alto risco. 2012. Disponível em: http://bvsms.saude.gov.br/bvs/publicacoes/manual_tecnico_gestacao_alto_risco.pdf. Acessado em: 15 de outubro de 2020.

7. CRIOLLO RA, et al. Embarazo ectópico cervical gemelar: presentácion de un caso inédito. Clínica e investigación en ginecología y obstetricia, 2016; 45(2).

8. De OTT D. A case of unilateral tubal twin gestation. Annales de Gynecologie et d'Obstetrique, 1891; $36: 304$.

9. DEDE M, et al. Unilateral Tubal Ectopic Twin Pregnancy. Taiwan J Obstetrics and Gynecology, 2008; 47(2).

10. DUTTA D. Obstetrics: including Perinatology and Contraception. 8th ed. New Delhi: Konar H, 2015; 274p.

11. ESCOLA UFRJ. Guia de Rotinas Assistenciais da Maternidade. Gemelidade. 2015. Disponível em: http://www.me.ufrj.br/images/pdfs/protocolos/obstetricia/gemelidade.pdf. Acessado em: 04 de novembro de 2020.

12. FEBRASGO. Guia de Gravidez Ectópica. 2018. Disponível em: http://www.grupoamigo.com.br/admin/wpcontent/uploads/2018/10/Gravidez-ectpica.pdf. Acessado em: 18 de novembro de 2020.

13. FEBRASGO. Manual de assistência pré-natal. 2014.2 Disponível em: https://portaldeboaspraticas.iff.fiocruz.br/biblioteca/manual-de-assistencia-pre-natal/. Acessado em: 20 de outubro de 2020.

14. FREITAS F, et al. Rotinas em Obstetrícia. 6th ed. Porto Alegre: Artmed; 2011; 767p.

15. GUELANDI M, et al. First reported case of preoperative ultrasonic diagnosis and laparoscopic treatment of unilateral, twin tubal pregnancy. Revue Francaise de Gynecologie et D'obstetrique, 1994; 89(3): 134-136.

16. HERRERA GM, et al. Embarazo ectópico del segundo trimestre. Revista Cubana de Ginecologia y Obstetricia, 2017; 43(3).

17. HOFFMAN BL, SCHORGE JO. Ginecologia de Willians. 2nd ed. Porto Alegre: ArtMed, 2014; 198p.

18.JUNIOR EJ, et al. Gravidez ectópica não rota - diagnóstico e tratamento: situação atual. Revista Brasileira de Ginecologia e Obstetrícia, 2008; 30(3).

19. MARTINS EF, et al. Causas múltiplas de mortalidade materna relacionada ao aborto no Estado de Minas Gerais. Caderno de Saúde Pública, 2017; 33(1).

20. PASCUAL MA, et al. Cervical ectopic twin pregnancy: diagnosis and conservative treatment. European Society of Human Reproduction and Enbryology, 2001; 16(3).

21. POSSER AO, et al. Gravidez ectópica tubária bilateral: relato de caso. RBGO, 1998; 20(6).

22. REZENDE J, MONTENEGRO CAB. Obstetrícia Fundamental. 13th ed. Rio de Janeiro: Guanabara Koogan, 2014; 423p.

23. VOHRA S, et al. Spontaneous live unilateral twin ectopic pregnancy - Acase presentation: ultrasound, 2014; 22(4).

24. ZUGAIB M. Obstetrícia. 4th ed. Rio de janeiro: Manole, 2019; 583p. 